\title{
Bringing light to the sirens of night: laryngoscopy in catathrenia during sleep
}

\section{To the Editors:}

Catathrenia is a rare sleep disorder characterised by groaning and prolonged expiration confined to sleep alternating with normal breathing that may occur at all sleep stages. After its first description in 1983 [1], catathrenia was recently introduced into the International Classification of Sleep Disorders [2]. Although the exact pathogenesis remains unknown, catathrenia has been considered a parasomnia. Important differential diagnoses include moaning during epileptic seizures, central sleep apnoea, sleep-related laryngospasm, expiratory snoring and stridor.
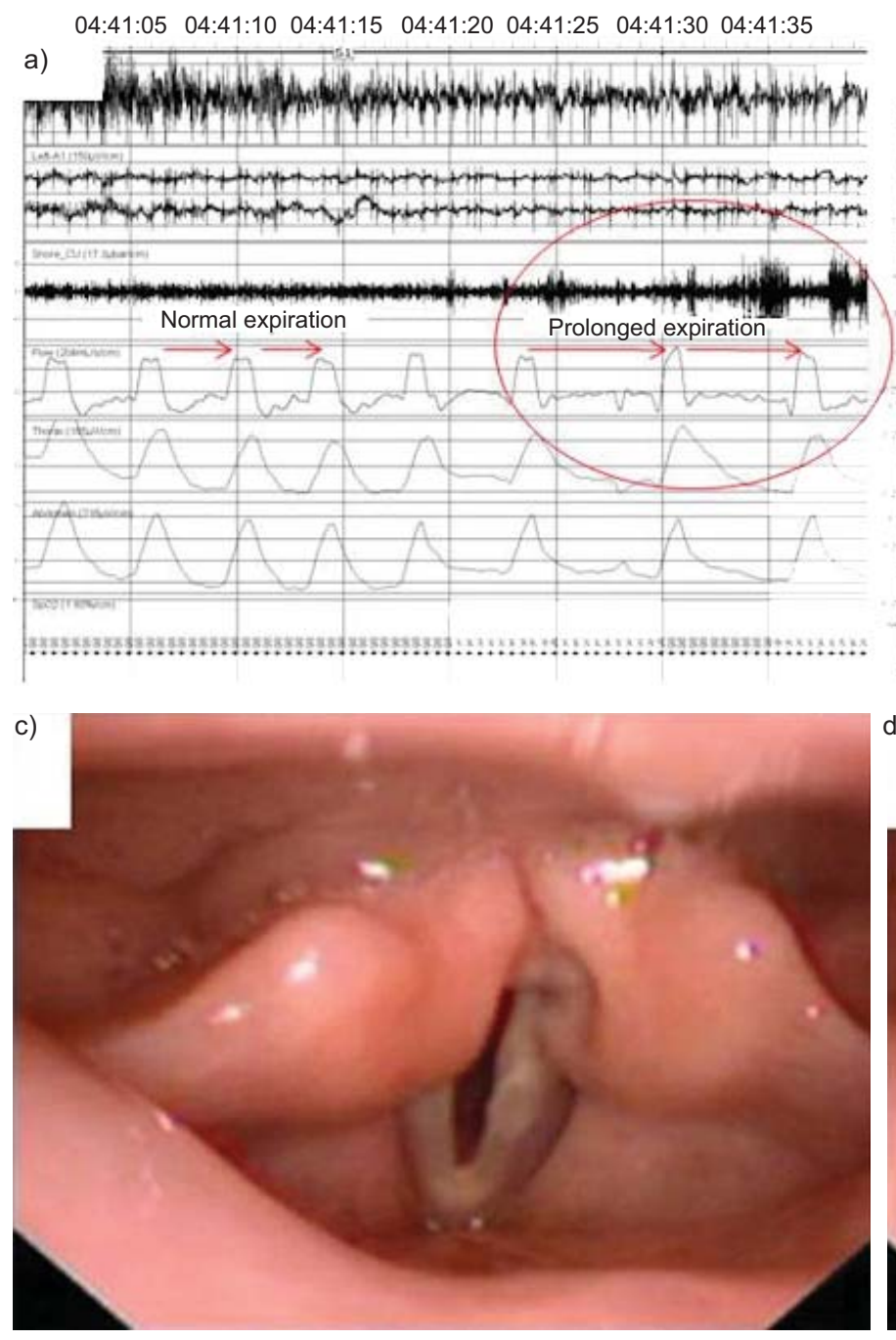

Most patients complain about social distress in their partnership, and only a minority suffers from excessive daytime sleepiness (EDS). Continuous positive airway pressure (CPAP) treatment has shown to be effective in some patients [3].

Several postulated theories exist on the underlying mechanism. OrtegA-Albas et al. [4] assumed the underlying mechanism was a dysfunction involving the post-inspiratory neurons that have inhibitory connections between them. This hypothesis only explains some of the clinical features of catathrenia, such as the lack of increase in intrathoracic pressure during
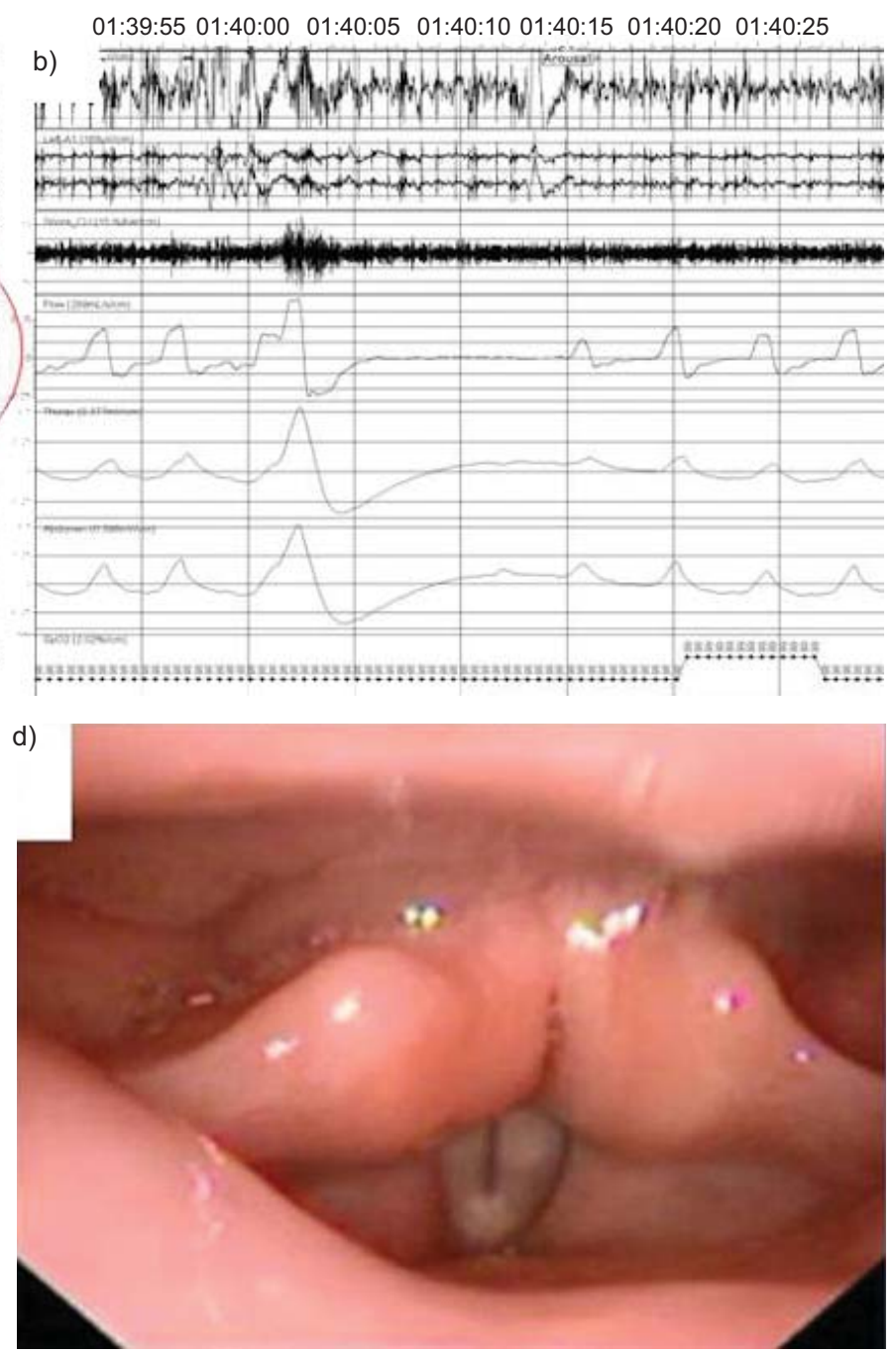

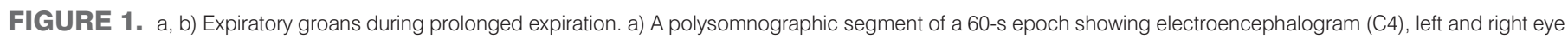

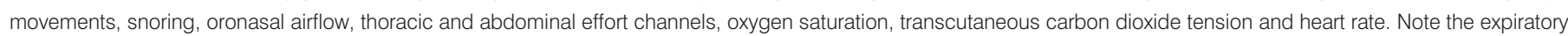

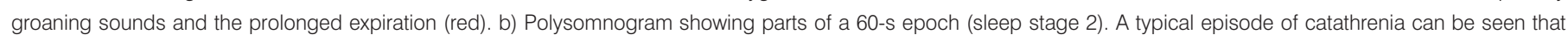

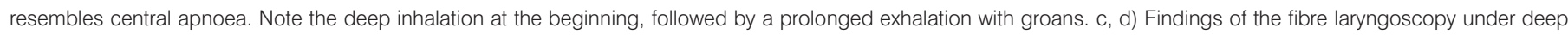

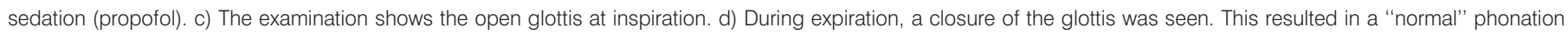
causing the groaning characteristic of catathrenia. 
expiration, but not necessarily the groaning. Although most patients show normal laryngoscopic findings during daytime examination, some authors suggest a partial closure of the glottis as the cause of the groaning, but this has never been proven.

We performed laryngoscopy during deep sedation using propofol in a 29-yr-old female without any comorbidites who was transferred for the evaluation of nocturnal groaning, EDS (Epworth Sleepiness Scale 17 out of 24 points), and exerciseinduced and occasional nocturnal dyspnoea, starting 15 yrs previously. A pulmonological examination, including bronchoscopy, revealed neither asthma nor any stenosis of the upper airways or the trachea.

The otorhinolaryngological examination showed a decreased mobility of the right vocal cord; there was a slight medial dislocation of the right arytenoid, but a cervical computed tomography scan revealed neither lysis nor dislocation or malformation of the arytenoids. Phonation was not affected.

A video polysomnogaphy showed an apnoea-hypopnoea index of 5 events $\cdot h^{-1}$; minimal oxygen saturation was $94 \%$. Snoring was not detectable but during major periods in rapid eye movement (REM) and non-REM sleep, groaning and prolonged expiration were observed (fig. 1a-b); diagnostic criteria for catathrenia were fulfilled and all major differential diagnoses were excluded.

Laryngoscopy under deep sedation showed the open glottis at inspiration, but during expiration, a subtotal closure of the glottis was seen, causing the groan that is typical for catathrenia (fig. 1c and d). During the groaning, the vocal cords perform an expiratory active adduction followed by a vibration; thus, the mechanism of the groaning is similar to a normal phonation.

Due to the EDS, we started treatment with nasal CPAP at a fixed pressure of $8 \mathrm{cmH}_{2} \mathrm{O}$, leading to a significant reduction in nocturnal groaning and improved wellbeing during the daytime.
This is the first description of a laryngoscopy in a sleeping patient with catathrenia. The active adduction of the vocal cords during expiration strongly supports the hypothesis of a phonation causing the groaning and, thus, of the parasomnic nature of catathrenia. As long as many unanswered questions remain, especially addressing the underlying pathophysiological mechanism of expiratory active closure of the vocal cords, attempting a CPAP trial in patients who may want treatment will be reasonable.

\section{S.R. Ott*, J. Hamacher* and E. Seifert ${ }^{\#}$}

*Dept of Respiratory and Sleep Medicine, and "Dept of Othorhinolaryngology, Inselspital, University Hospital and University of Berne, Berne, Switzerland.

Correspondence: S.R. Ott, Dept of Respiratory and Sleep Medicine, Inselspital, University Hospital Berne, CH-3010 Berne, Switzerland. E-mail: Sebastian.ott@insel.ch

Statement of Interest: None declared.

\section{REFERENCES}

1 De Roek J, van Hoof E, Cluydts R. Sleep-related expiratory groaning. A case report. J Sleep Res 1983; 12: 237.

2 American Academy of Sleep Medicine. International Classification of Sleep Disorders, 2nd Edn: Diagnostic and Coding Manual. Westchester, American Academy of Sleep Medicine, 2005.

3 Guilleminault C, Hagen CC, Khaia AM. Catathrenia: parasomnia or uncommon feature of sleep disordered breathing? Sleep 2008; 31: 132-139.

4 Ortega-Albas JJ, Diaz JR, Serrano AL, et al. Continous positive airway pressure as a treatment for catathrenia (nocturnal groaning). Neurology 2006; 67: 1103.

\section{The search for autoantibodies against elastin, collagen and decorin in COPD}

\section{To the Editors:}

Several studies indicate that an autoimmune response may underlie the pathogenesis of chronic obstructive pulmonary disease (COPD). A high prevalence of anti-Hep-2 epithelial cell autoantibodies has been shown in patients with COPD [1, 2], as well as autoantibodies against airway epithelial cells [1], endothelial cells [3], lung elastin [4], several immunogenic peptides [5] and cytokeratin 18 [6]. The most convincing evidence for an autoimmune response in COPD was provided by LeE et al. [4]. They showed a specific autoantibody and a specific T-cell response against elastin in COPD patients, as well as increased numbers of anti-elastin antibody-producing cells in the lungs of COPD patients. Recent studies [7, 8], however, could not confirm such an anti-elastin autoantibody response. COTTIN et al. [7] found no evidence for an anti-elastin autoantibody response in patients with combined pulmonary fibrosis and emphysema. Additionally, GREENE et al. [8] investigated the presence of autoantibodies against elastin- and collagen-derived proline-glycine-proline in patients with COPD and $\alpha_{1}$-antitrypsin deficiency, and also did not observe increased autoantibody levels.

We assessed the presence of autoantibodies against elastin, collagen and decorin in serum of COPD patients and healthy controls. In addition, and as an extension of previous studies, we included equal numbers of current, ex- and never-smokers, in order to investigate the effect of the smoking status on 